\title{
Análisis de calidad percibida y expectativas de pacientes en el proceso asistencial de diálisis
}

\section{Patients' opinions and expectations about the dialysis care process}

\author{
M.A. Prieto, M.J. Escudero, A. Suess, J.C. March, A. Ruiz, A. Danet
}

\section{RESUMEN}

Fundamento. Conocer en profundidad las vivencias y necesidades de pacientes en diálisis con el objetivo de identificar los puntos críticos del proceso de atención y elaborar propuestas de mejora.

Métodos. Estudio cualitativo realizado a partir de 22 entrevistas semiestructuradas con pacientes en hemodiálisis y diálisis peritoneal, del Servicio Andaluz de Salud. Análisis de discurso, utilización del modelo SERVQUAL. Triangulación de resultados.

Resultados. Los pacientes describen la fase de diagnóstico como el momento más duro por la necesidad de aceptación de la enfermedad. Durante la hemodiálisis, se constata tanto reacciones de adaptación positiva como la percepción de una disminución de la calidad de vida. La técnica de diálisis peritoneal se valora positivamente por permitir una mayor independencia, pese a requerir una mayor responsabilidad de autocuidado. El contacto con una asociación de afectados o la disposición de un servicio de atención psicológica se valoran como elementos de ayuda en el proceso. Respecto a diferentes dimensiones del modelo SERVQUAL, se valora el trato humano y la competencia profesional. Los puntos críticos son: la falta de coordinación, el mal funcionamiento del transporte y la falta de transparencia en la gestión de las listas de espera. Se señalan deficiencias en el trato a cuidadores informales y en el nivel de conocimiento de profesionales de otras áreas diferentes a de la Nefrología.

Conclusiones. Desde la perspectiva de los pacientes nefrológicos, la atención a los aspectos psicosociales, la mejora de aspectos organizacionales como el transporte, así como una mayor atención a los cuidadores informales se identifican como propuestas prioritarias para una mejora del proceso de diálisis.

Palabras clave. Diálisis. Pacientes.

\begin{abstract}
Background. To determine the experiences and needs of patients on dialysis, in order to identify critical points of the care process and develop proposals for improvement.
\end{abstract}

Methods. Qualitative study using semistructured interviews with 22 patients on hemodialysis and peritoneal dialysis, from the Andalusian Health Service. Discourse analysis, using the SERVQUAL model. Triangulation of results.

Results. The diagnostic stage is described as the hardest moment as it requires acceptance of the disease During hemodialysis, we see both positive adaptation and the perception of a diminished quality of life. The technique of peritoneal dialysis is evaluated positively, enabling greater independence, despite requiring more responsibility for self care. The contact with patients'organizations or the provision of a counseling service are valued as an aid in the process. With respect to different dimensions of the SERVQUAL model, human treatment and professional competence are valued. The critical points are lack of coordination, malfunctioning of transportation and lack of transparency in the management of waiting lists. Shortcomings in dealing with informal caregivers and the level of knowledge of professionals from areas other than Nephrology, also appear as deficiencies.

Conclusions. The main proposals for improving the dialysis process are: attention to psychosocial aspects, the improvement of organizational aspects such as transport, and greater attention to informal caregivers.

Keywords. Dialysis. Patients. Health Services.

An. Sist. Sanit. Navar. 2011; 34 (1): 21-31

\section{Escuela Andaluza de Salud Pública}

Recepción: 5 de octubre de 2010

Aceptación provisional: 19 de noviembre de 2010

Aceptación definitiva: 20 de diciembre de 2010

\section{Correspondencia:}

Alina Danet Danet

Escuela Andaluza de Salud Pública

Cuesta del Observatorio, 4

18011 Granada. España

E-mail: alina.danet.easp@juntadeandalucia.es 


\section{INTRODUCCIÓN}

En las sociedades contemporáneas, la atención a enfermedades crónicas adquiere una relevancia cada vez mayor y plantea nuevos desafíos a la planificación de los procesos sanitarios ${ }^{1}$. Aparte de indicadores como competencia técnica o rapidez de respuesta, el término "calidad de vida" aparece como un concepto relevante ${ }^{2}$. En este sentido, se amplía el foco de una atención centrada en la competencia profesional y rapidez de resolución a la inclusión de factores como el trato, la consideración de aspectos emocionales y psicosociales y la equidad en el acceso. La inclusión de la perspectiva de los pacientes en el proceso de diseño e implantación se considera un elemento relevante en una atención sanitaria que comprende al usuario como "centro del sistema" ${ }^{3,4}$.

El proceso asistencial de diálisis requiere una atención sanitaria prolongada y continuada. En la bibliografía especializada, se encuentran diferentes trabajos $^{5-19}$ que analizan la interrelación entre calidad de vida percibida y tratamiento nefrológico, estableciendo comparaciones respecto a la calidad de vida en diferentes modalidades de diálisis (hemodiálisis hospitalaria, diálisis peritoneal $)^{7,13}$ y entre diálisis y transplante $^{5,8,19}$. Algunos autore ${ }^{20-22}$ centran su análisis en la presencia de depresiones u otras alteraciones del estado emocional de los pacientes en diálisis, así como la influencia de la duración del tratamiento en la vivencia psicosocial ${ }^{7,16,23}$. Las diferencias en la calidad de vida según procedencia cultural $^{24} \mathrm{o}$ edad ${ }^{8}$, la percepción de dolor durante el tratamiento ${ }^{25}$, así como la vivencia de los cuidadores y familiares ${ }^{26-29}$ constituyen otros puntos de interés. En algunos textos ${ }^{14,30-40}$, se puede encontrar un análisis de diferentes momentos del proceso asistencial, así como de distintos modelos organizacionales de atención.

Desde la consideración de la metodología cualitativa como herramienta válida para conocer en profundidad las opiniones, sentimientos y expectativas de los pacientes $^{41}$, en el presente estudio se han realizado entrevistas semiestructuradas con pacientes. Aparte de preguntas enfocadas a conocer la vivencia emocional y psicosocial de la enfermedad y del tratamiento, la investigación se centra en el conocimiento de fortalezas, debilidades y propuestas de mejora en diferentes momentos del proceso asistencial.

\section{MÉTODOS}

Tras obtener la aprobación del Comité de Investigación de la Escuela Andaluza de Salud Pública, se inició el estudio, realizando un muestreo intencional de $22 \mathrm{pa}-$ cientes: 18 pacientes en tratamiento con hemodiálisis, 1 paciente en diálisis peritoneal y 3 pacientes en diálisis peritoneal con cicladora. Se realizaron por tanto 22 entrevistas semiestructuradas. Todas las personas entrevistadas habían sido tratadas por el Sistema Sanitario Público de Andalucía, y fueron contactadas a través de hospitales o centros periféricos de diálisis en los que estaban recibiendo tratamiento. Se entrevistó a mujeres y hombres de edades entre los 26 y 79 años que provenían tanto del medio rural como urbano. Asimismo, se intentó recoger la experiencia de situaciones diferentes respecto al tiempo de tratamiento con la diálisis (de pocos meses a 15 años), así como respecto al momento en el proceso asistencial (personas en lista de espera para un transplante, personas transplantadas y pacientes sin posibilidad de transplante). Como criterio de inclusión, se definió ser paciente en proceso de hemodiálisis o diálisis peritoneal, estableciendo como criterio de exclusión tener un estado de salud física o psíquica demasiado delicado para participar en proceso de entrevista, pudiendo poner en peligro la integridad física o psíquica del paciente.

Las entrevistas se realizaron en centros de diálisis y en el domicilio de los pacientes, en varias provincias de la Comunidad Autónoma andaluza. Todos los participantes dieron su consentimiento informado, que fue verbal y se grabó al comenzar las entrevistas.

Después de transcribir las entrevistas, se realizó un análisis de contenido triangu- 
lado por diferentes miembros del equipo investigador, estructurando la información obtenida siguiendo las dimensiones del modelo SERVQUAL (accesibilidad, trato, comunicación, competencia, capacidad de respuesta, tangibilidad) ${ }^{42}$ (Tabla 1$)$. El proceso de triangulación se ha realizado entre las personas investigadoras del estudio y con los resultados de otros estudios de la bibliografía disponible.

Tabla 1. Dimensiones de análisis

\begin{tabular}{ll}
\hline \multicolumn{1}{c}{ Dimensión } & \multicolumn{1}{c}{ Categorías } \\
\hline \multirow{2}{*}{ Tipo de tratamiento } & - Hemodiálisis \\
& - Diálisis peritoneal \\
& - Diálisis peritoneal con cicladora \\
\hline Momento en el proceso asistencial & - En espera de transplante \\
& - Transplantados \\
& - Sin posibilidad de transplante \\
\hline Duración de la hemodiálisis & - Primeros meses del proceso de hemodiálisis \\
& - Más de un año en proceso de hemodiálisis \\
\hline Características sociodemográficas & - Sexo \\
& - Edad \\
& - Lugar de residencia (urbano / rural) \\
\hline Aspectos del proceso asistencial según el modelo & - Accesibilidad \\
SERVQUA & - Trato \\
& - Comunicación \\
& - Capacidad de respuesta \\
\end{tabular}

\section{RESULTADOS}

\section{Vivencia general de la enfermedad y del tratamiento}

En los relatos de las personas entrevistadas, se pueden identificar experiencias diferenciadas según el momento en el proceso asistencial y según la técnica de diálisis (hemodiálisis o diálisis peritoneal).

Según los participantes del estudio, el proceso asistencial se suele iniciar por la percepción de síntomas y un primer contacto con el centro de Atención Primaria o por una detección del problema en un ingreso hospitalario ajeno a la enfermedad nefrológica. Ante la sospecha diagnóstica, comienza un proceso de derivación y realización de pruebas por consultas externas de Atención Especializada. En consecuencia del carácter difuso de la sintomatología, en algunos casos el proceso de detección, se dilata en el tiempo. Después de establecerse el diagnóstico, no todos los pacientes inician enseguida un tratamiento de diálisis, produciéndose en algunos casos periodos prolongados de latencia o de tratamiento farmacológico. Algunos pacientes combinan contactos con la asistencia pública y privada, especialmente en la primera fase de detección y diagnóstico.

Es el momento en el que se comunica la necesidad de iniciar un tratamiento de diálisis el que los pacientes viven con un mayor grado de angustia. Los participantes nombran como un momento de impacto especial el primer contacto con la sala de hemodiálisis. En caso de la diálisis peritoneal, la angustia se centra en la responsabilidad del manejo correcto de la maquinaria y del mantenimiento de la higiene. A modo general, los entrevistados describen la dificultad de asumir una dependencia prolongada de un tratamiento periódico y las consecuencias que tendrá para su vida personal y profesional. 
Anexo 1. Valoración del tratamiento por tipo de diálisis

\begin{tabular}{|c|c|}
\hline Tipo de diálisis & Experiencias \\
\hline Hemodiálisis & $\begin{array}{l}\text { Primer contacto } \\
\text { "Lo que yo vi allí de tanto enfermo yo no, yo no, yo decía, si Dios me va a tener en esta má- } \\
\text { quina muchos años que me muera antes, porque eso, como alli se ve de todo, no porque estén } \\
\text { mal atendidos..., sino porque, pues, hay unos mejores, unos peores, unos más jóvenes, unos } \\
\text { más viejos, entonces, tú después de que estâs desmoralizada te ves en este sitio". } \\
\text { "Se me cayó el cielo encima... nerviosa, llorando mucho. (...) Yo pensaba que esto, toda la } \\
\text { vida tenía que estar con tratamiento y que nunca tendría que llegar a esto, porque yo no lo } \\
\text { conocía. Estâs tranquila en tu casa, tu vida, tu marido, tu casa, tu niña y ahora estar asî, pues } \\
\text { te cambia... la vida te cambia totalmente”. } \\
\text { Vivencia de la hemodiálisis } \\
\text { "Yo tengo una diálisis muy buena. Ah, ya me habrás visto dormir. Siempre estoy durmiendo } \\
\text { las cuatro horas". } \\
\text { "La máquina... me mareo un poquillo, me dan calambres". } \\
\text { Calidad de vida } \\
\text { "Yo siempre era una persona que viajaba mucho (...), pues bien, y ahora ya te ha cambiado } \\
\text { la vida, ahora ya... estás atada a una máquina, ¿no?" } \\
\text { "En calidad de vida, yo al menos ahora puedo comer de todo un poquito pero como, y antes } \\
\text { era, no podía, no sabía qué tomar, en fin, o sea, que prefiero la diálisis porque al menos me } \\
\text { llevan controlado en todo". } \\
\text { Síntomas depresivos } \\
\text { "Porque hay momentos que yo, pues, pues casi caigo en una depresión porque yo me encuen- } \\
\text { tro con cincuenta y ocho años que tengo, invalida y dependiendo de los demás, y a mí eso } \\
\text { me duele y lloro". }\end{array}$ \\
\hline Diálisis peritoneal & $\begin{array}{l}\text { Comodidad } \\
\text { "La verdad es que estoy contenta, luego, la comodidad de hacértelo en tu casa, el no tener } \\
\text { que... que estar un día sí y otro no esperando allí a que te puedas meter en la máquina, a estar } \\
\text { cuatro horas allí, a..., ¿entiendes?, que para mí es muy cómodo conectarme todas las noches } \\
\text { a la máquina, que yo me acuesto a dormir tranquilamente". } \\
\text { Autonomía } \\
\text { "Sí, es que yo no aguanto que me pinchen, ir un día sí y un día no a que me pinchen, además } \\
\text { esto da, da más autonomía, hasta para poder estar trabajando". } \\
\text { Responsabilidad } \\
\text { "Pues, yo ya sabiendo... siempre teniendo la limpieza que hay que tener. Con todo, con la } \\
\text { casa, con el cuerpo y con todo, con todo porque es que esto tiene un defecto, que coges unas } \\
\text { infecciones, jpuf!, que son muy grandes". }\end{array}$ \\
\hline
\end{tabular}

Después de un tiempo de tratamiento, la mayoría de las personas entrevistadas relatan haber vivido un proceso de adaptación y asunción de la enfermedad, en la que la presencia de "la máquina" pasa de vivirse como una amenaza a percibirse como una ayuda y garantía. Los entrevistados destacan la importancia de recibir apoyo y comprensión por parte de los profesionales sanitarios especialmente en esta fase del proceso.

Respecto al proceso de hemodiálisis, se observan vivencias diferenciadas. Mientras algunas personas experimentan el tiempo de conexión a la máquina con tranquilidad, que les permite descansar, leer o dormir, otros pacientes dicen percibir dolores y mareos, así como haber tenido problemas con las fístulas. Algunos pacientes, ante todo mujeres, señalan que les angustia el tiempo que tienen que pasar conectadas a la máquina por tener que dejar desatendidas otras obligaciones y responsabilidades. La comunicación con los otros pacientes en la sala alivia la percepción de tiempo de conexión a la máquina. Asimismo, se 
percibe con agrado la posibilidad de cambiar de centro para poder continuar el proceso de diálisis en el lugar de vacaciones.

En la mayoría de los pacientes, incluso en aquellos que perciben la hemodiálisis como elemento de garantía en el proceso de enfermedad, se puede observar un aumento de la desmotivación y angustia conforme se va prolongado el tiempo de su aplicación. Como inconvenientes se nombra la restricción en la libertad de movimiento y el control en la alimentación e ingesta de líquidos que requiere. Una preocupación añadida constituyen los efectos secundarios a largo plazo del tratamiento de hemodiálisis en el organismo.

Ante la percepción de una disminución de calidad de vida, se observan diferentes grados de adaptación emocional a la situación que llegan desde intentos de asunción y resignificación positiva hasta la presencia de estados depresivos.

Respecto a la diálisis peritoneal, tanto con el método convencional como con cicladora, se destacan como ventajas la posibilidad de realizarla en casa, poder adaptar su realización a los horarios propios, poder irse de vacaciones sin dependencia de un centro de diálisis y no tener que cuidar la alimentación y la ingesta de líquido. La mayoría de los pacientes dicen ser capaces de dormir durante la aplicación, mientras algunas personas señalan experimentar dolores. Una preocupación añadida es la limpieza adecuada del dispositivo para evitar infecciones. Los pacientes dicen sentir una cierta intranquilidad, especialmente en los primeros días, por tratarse de un tratamiento que requiere una responsabilidad propia, hasta poder comprobar el funcionamiento correcto de la máquina (Anexo 1).

En ambos grupos de pacientes, en independencia de la técnica usada, se nombra como una preocupación central los tiempos de espera prolongados para un transplante, así como el riesgo de rechazo y necesidad de un nuevo transplante. No disponer de información sobre la gestión de las listas de espera se identifica como un factor que puede contribuir a una sensación de angustia en la vivencia del tiempo de espera.

Además, los entrevistados destacan la relevancia de la dimensión social de la enfermedad nefrológica, por tratarse de una enfermedad crónica que requiere un tratamiento constante y que, según su percepción, no está libre de un cierto estigma social que puede afectar a la relación con el entorno social o laboral. Según su experiencia, la dependencia del tratamiento de diálisis supone una modificación de la vida personal y familiar no sólo de la persona afectada, sino también de los cuidadores familiares, para quienes los participantes del estudio reclaman una mayor atención por parte del Sistema Sanitario Público (Tabla 2).

Tabla 2. Aspectos sociales

\footnotetext{
Influencia en la vida familiar

"Yo tengo dos niños y tengo que dejar mi casa abandonada: mi marido, mi casa, mis niños".

Influencia en la vida laboral

"Es muy difícil que a una persona con una enfermedad crónica te contraten, vamos y las relaciones de parejas, pues igual, no es fácil que una persona quiera acarrear, entre comillas, contigo".

Estigma social

"Hay enfermedades que presume la gente, presumen como si fueran elegantes, como si fuera de, de gente bien y hay otras cosas que se avergüenzan de decirlo, ¿por qué?, porque eso es así. Ésta es de las que nadie quiere que se enteren, ésta es la que nadie quiere que se enteren y lo primero que piensan, instintivamente, es menos mal que no lo tengo yo".

Vivencia de los cuidadores informales

"Yo creo que en el Servicio de Asistencia Sanitaria debería haber ayuda psicológica para los familiares, porque es que estamos muy desamparados".
} 
Como estrategias relevantes para una mejora de la calidad de vida y aceptación de la enfermedad se nombra el servicio de apoyo psicológico y el contacto con una asociación de afectados. Asimismo, se menciona la importancia de poder disponer de ayudas para sobrellevar los aspectos sociales de la enfermedad.

\section{DIMENSIONES DEL PROCESO ASISTENCIAL}

Aparte de describir la vivencia general de la enfermedad y del tratamiento recibido, los entrevistados mencionan diferentes aspectos del proceso asistencial que se analizarán siguiendo las dimensiones del SERVQUAL ${ }^{42}$.

\section{Accesibilidad}

La mayoría de los entrevistados coinciden en una evaluación positiva respecto a la accesibilidad, sintiéndose tratados como "pacientes preferentes" por el Sistema Sanitario Público. La posibilidad de realizar consultas y pruebas en los mismos centros de diálisis, sin tener que acudir a la consulta del especialista, se percibe como una ventaja. Los pacientes en diálisis peritoneal valoran positivamente la posibilidad de contar con personal técnico de la empresa que facilita los instrumentos para consultar dudas sobre su funcionamiento. A su vez, algunos pacientes señalan que la experiencia de dificultad de acceso a los especialistas les llevó a un uso del "atajo" a través de los servicios de urgencias.

\section{Cortesía}

En general, se constata una buena calidad de trato por parte de los profesionales. Por el carácter de enfermedad crónica que requiere tratamiento continuado, los pacientes nefrológicos entrevistados otorgan un alto nivel de importancia al trato humano, a la capacidad de empatía y a la atención a aspectos emocionales. A diferencia de una evaluación positiva del trato recibido como pacientes, éstos mismos señalan un menor nivel de atención, especialmente por parte del personal administrativo, hacia los familiares que les acompañan durante las estancias hospitalarias, demandando una mejora del trato y de la atención a sus necesidades específicas.

\section{Comunicación}

Los pacientes coinciden en la percepción de haber obtenido información adecuada sobre la enfermedad y sus tratamientos. Destacan la relevancia de un alto nivel de información y la posibilidad de participar en los procesos de toma de decisión. Asimismo, valoran positivamente el grado y la calidad de información hacia familiares y cuidadores. A la vez, identifican algunas lagunas informativas sobre el proceso de transplante y el funcionamiento de la lista de espera y los criterios de selección, demandando una mayor información sobre estos aspectos.

En general, los entrevistados dicen haber tenido oportunidad de expresar sus dudas. Sin embargo, también se mencionan algunas dificultades en plantear preguntas, por propia inseguridad, o por miedo de no entender la respuesta del médico. La práctica de un intercambio de información entre pacientes se percibe como una ayuda para despejar inquietudes sobre el autocuidado y la seguridad de la maquinaria.

\section{Competencia}

Se puede observar un alto grado de confianza en la competencia profesional de los especialistas. Sin embargo, se constata una falta de conocimientos sobre las características específicas de la enfermedad y un mayor riesgo de errores en profesionales sanitarios ajenos a la especialidad de Nefrología. Los pacientes responden a estas experiencias con un aumento del grado de alerta, asumiendo parte de la responsabilidad de control.

Se expresa la expectativa de una mayor formación de los profesionales de otras áreas sanitarias sobre las necesidades de los pacientes nefrológicos, así como una 
mayor dotación de recursos en la investigación, para facilitar un avance en el estado de conocimiento sobre la enfermedad y sus posibilidades de tratamiento.

\section{Capacidad de respuesta}

Los pacientes entrevistados critican el alto grado de variabilidad en la capacidad de respuesta y tiempos de espera. Mientras algunos pacientes no han encontrado dificultades en el proceso de citación, otros destacan tiempos de espera prolongados en el proceso de realización de pruebas, así como una falta de coordinación entre el momento del ingreso hospitalario y la intervención quirúrgica. En cambio, se observa un mayor grado de satisfacción respecto a la periodicidad de las citas de seguimiento.

Anexo 2. Valoración de la calidad de atención por dimensiones del modelo SERVQUAL

\begin{tabular}{cl}
\hline $\begin{array}{c}\text { Dimensiones del } \\
\text { SERVQUAL }\end{array}$ & Experiencias \\
\hline \multirow{2}{*}{ Accesibilidad } & $\begin{array}{l}\text { Facilidad en el acceso } \\
\text { "Te acercas enseguida y te atienden enseguida, no tienes problemas de ningún tipo, cualquier } \\
\text { momento, cualquier cosa, llegas y te atienden enseguida, o sea, en eso no hay problemas". }\end{array}$ \\
\hline & Trato recibido \\
& "Y, luego, la atención, buenísima, pero por todas las partes, tanto por las empresas que nos \\
& sirven los productos, como por el hospital, la atención maravillosa". \\
& "Estấn pendientes de ti, si necesitas algo no tienes nada más que mirarlos, ellos estân aquí en- \\
& cima... pero... los médicos, los especialistas, no, no he tenido con ninguno, ningún problema, \\
& al contrario, muy bien con ellos".
\end{tabular}

\section{Grado de información recibida}

"Sí, a mí me han dado información sobre los sistemas de diálisis, me han dado información sobre trasplante y me han dado, o sea, yo, yo no puedo decir que tengo quejas".

Comunicación ¿ ¿Tiene, usted, información sobre cómo funciona la lista de espera?"

"No, yo ya no".

"Y, ¿le gustaría saberlo o..."?

"Pues sî..., me gustaría porque a ver quién trasplantan antes, a ver quién trasplantan después".

\begin{tabular}{ll}
\hline & Especialistas de Nefrología: \\
& "Tengo buenos médicos... tanto el doctor, como el doctor, son muy buenos". \\
Competencia & Otras áreas de atención sanitaria: \\
& "Yo tengo por norma, de cuando yo voy a otro médico que no sea del riñón, llevo mi lista de \\
& mis medicinas y lo pongo en la mesa para que las vea".
\end{tabular}

\section{Coordinación:}

Capacidad de Me parece horroroso, vamos, que tengas que entrar una semana antes, previendo que el respuesta quirófano va a estar una semana después (...), porque yo me he sentido muy mal allí ingresado, vamos, para mi ingresarme es la cárcel, vamos. Pero que si hubiese un poquito más de coordinación..."

\section{Percepción de seguridad:}

Seguridad "Me aporta seguridad, sí, seguridad, saber que estoy en buenas manos, vamos, saber que cualquier problema que tengas incluso psicológico, que es algo que te afecte no sólo fisicamente, sino que tú le preguntes al médico me pasa esto y me preocupa esto, enseguida te atienden".

\section{Transporte:}

"Lo que menos me gusta es la ambulancia, porque es un poquillo... porque venimos muy hacinados en ella, ¿no?, como van recogiendo a gente, venimos mucha gente".

Tangibilidad "Y qué tal en el taxi?"

"Muy bien. Me gusta más que el ambulancia, porque en las ambulancias van todos... y hacen mucho recorrido para recoger a todos..." 


\section{Seguridad}

Para los pacientes entrevistados, la percepción de seguridad no sólo está relacionada con aspectos exteriores como el mantenimiento adecuado de la maquinaria, sino también con factores como la confianza en la competencia profesional, la disponibilidad de una información exhaustiva, la facilidad en el acceso a los profesionales para consultar dudas y la posibilidad de participar en los procesos de toma de decisión.

\section{Tangibilidad}

Los pacientes entrevistados coinciden en una valoración general positiva en aspectos de la atención recibida, considerando un alto nivel de recursos materiales disponibles. En los ingresos hospitalarios, se critica la incomodidad de las camas, tanto para los pacientes como las butacas para los acompañantes. Respecto a los tratamientos de hemodiálisis, se expresa una cierta preocupación respecto al estado de las máquinas, pidiendo su renovación y revisión continuada.

Sin embargo, como punto más débil en el proceso de hemodiálisis se identifica el transporte. Mientras las personas a las que el Servicio Andaluz de Salud les proporciona transporte en taxi están satisfechas con el servicio, los pacientes trasladados en ambulancias expresan un mayor grado de insatisfacción, criticando los tiempos prolongados de traslado y la frecuencia de retrasos. Asimismo, se percibe una falta de transparencia en los criterios de asignación del modo de transporte.

Las personas que están en diálisis peritoneal manifiestan un alto grado de satisfacción respecto a la maquinaria y los servicios de soporte técnico facilitados por la empresa dispensadora (Anexo 2).

\section{DISCUSIÓN}

La metodología cualitativa facilitó el conocimiento en profundidad de las vivencias y necesidades de los pacientes y per- mitió la identificación tanto de aspectos coincidentes con otros estudios del ámbito internacional, como de elementos propios del contexto sanitario estudiado.

Los pacientes nefrológicos entrevistados se perciben como "pacientes preferentes" dentro del Sistema Sanitario Público. Elogian el trato recibido por los facultativos y el personal de enfermería y muestran un alto grado de confianza en los especialistas que les tratan y en la calidad de las instalaciones técnicas.

Esta imagen positiva se matiza al hablar de los aspectos organizacionales: se constata una falta de transparencia en la gestión de las listas de espera para el transplante, problemas en la organización de los transportes, desigualdad en la atención recibida y escasez de coordinación intraservicios e intraniveles. Estos resultados coinciden con estudios anteriores ${ }^{14,30-32,35,36,38}$ en los que se identificaron aspectos organizacionales como la accesibilidad al centro de diálisis, la continuidad asistencial, la organización interna de la unidad o la coordinación con otros servicios y niveles asistenciales como puntos de mejora prioritarios. En este sentido, desde la perspectiva de la Agencia de Calidad del Sistema Nacional de Salud, la coordinación entre Atención Primaria y Especializada se identifica como "clave para mejorar la calidad de la Nefrología" 31 . En un estudio sobre la satisfacción de los pacientes en hemodiálisis realizado en el ámbito español ${ }^{14}$, se identificaron como aspectos de mayor influencia en el grado de satisfacción factores organizacionales como la puntualidad de las sesiones, la rapidez de respuesta a necesidades específicas y el tiempo de espera para ser atendido por el médico, así como el trato recibido por el personal de Enfermería. La importancia de una mejora de la planificación del proceso asistencial nefrológico, especialmente en la atención de pacientes con pluripatologías, se confirma en otro estudio $^{38}$ en el que se hace hincapié en la necesidad de un establecimiento de líneas de trabajo definidos, una reducción de la burocracia y mejora del trabajo en equipo. Diferentes autores constatan la existencia 
de un alto grado de variabilidad en la accesibilidad y atención según el centro hospitalario ${ }^{18,32}$ y según la procedencia cultural del paciente ${ }^{24}$ Los resultados del presente estudio coinciden con esta valoración de una falta de igualdad y homogeneidad en la atención sanitaria recibida.

En los estudios revisados, también se nombran estrategias de mejora, como la creación de un centro de diálisis dentro del hospital de referencia para subsanar los problemas de transporte ${ }^{36}$, o la introducción de la telemedicina en la diálisis peritoneal para mejorar la continuidad de los cuidados $^{39}$.

En la bibliografía revisada, se hace referencia a la relevancia de la información y de la oportunidad de participación en los procesos de decisión ${ }^{27,33,37}$. En el ámbito andaluz, Cirera y Reina ${ }^{37}$ evaluaron positivamente la calidad de información que ofrece el personal de enfermería de los centros periféricos de hemodiálisis a sus pacientes, así como la existencia de protocolos de información. En el presente estudio, se comparte esta valoración positiva de la calidad de información sobre la diálisis, a la vez de criticarse la ausencia de información respecto a la gestión de las listas de espera. Al igual que los pacientes entrevistados en el marco del estudio presentado, en la bibliografía revisada se señala la importancia de una participación de los pacientes en los procesos de toma de decisión sobre el tratamiento ${ }^{33}$. En caso de una toma de decisión por sustitución, se alerta el riesgo de que la decisión tomada refleje más las preferencias de los representantes que de los pacientes, razón por la que se apoya la implantación de procesos de planificación avanzada de los cuidados $^{6,27}$.

Según los testimonios de los pacientes nefrológicos entrevistados, la percepción de la seguridad no está limitada a factores técnicos (como el funcionamiento correcto de la maquinaria), sino que incluye aspectos como una información exhaustiva, la facilidad de plantear preguntas y una posibilidad de participar en los procesos de decisión, confirmando un concepto multi- dimensional de "seguridad del paciente" analizado en otros trabajos ${ }^{43}$.

Aparte de una evaluación de las áreas de mejora en diferentes fases del proceso asistencial, en los trabajos revisados se encuentran numerosos estudios ${ }^{7-20,23}$ sobre la influencia de la enfermedad y de los diferentes tipos de tratamiento en la calidad de vida de los pacientes. Coincidiendo con los relatos de los pacientes entrevistados en el presente estudio, se constata, en algunos casos, el empeoramiento del estado psicosocial y de las necesidades de cuidado conforme se va prolongando el tratamiento de hemodiálisis ${ }^{15,16,23}$. Diferentes autores ${ }^{12,20}$ señalan la frecuencia de estados depresivos en pacientes en diálisis y señalan la relevancia de un trato de aspectos psicosociales en la atención ${ }^{12}$. Rodríguez, Hernández, Gutiérrez y col ${ }^{25}$ evalúan la percepción de dolor durante la hemodiálisis, coincidiendo con la vivencia de algunos entrevistados de esta investigación sobre la presencia de sensaciones de malestar físico durante la diálisis. Lies-Smith ${ }^{21}$ señala la falta de estudios sobre la fase de pre-diálisis, identificando, en un estudio cualitativo, la presencia de sensaciones de fatalidad y desesperanza en los pacientes, un resultado confirmado en los discursos de los entrevistados de la presente investigación que identifican el momento después del diagnóstico como un momento difícil dentro del proceso asistencial en el que el trato humano por parte de los profesionales sanitarios se hace especialmente importante. El miedo a la responsabilidad de autocuidado en el cambio de hemodiálisis a diálisis peritoneal que mencionan los participantes de esta investigación se describe también en estudios anteriores ${ }^{9}$ en los que se destaca la importancia de programas educativos. Algunos trabajos ${ }^{28,29}$ analizan la carga emocional de los familiares y cuidadores informales, un aspecto nombrado también por los participantes de la presente investigación. Mientras en estudios previos $^{26}$ se señala la mejora de calidad de vida en los pacientes transplantados frente a los pacientes en proceso de diálisis, en el presente estudio los entrevistados, a la vez de manifestar el deseo de una reducción de 
las listas de espera, relatan la vivencia de dificultades en el proceso de transplante, debido a experiencias de rechazo e intervenciones reiteradas.

Para mejorar la calidad de vida percibida del paciente nefrológico, en la bibliografía revisada se resalta la relevancia de la relación médico-paciente ${ }^{17}$ y enfermeropaciente ${ }^{9}$, así como la conveniencia de una introducción de programas de apoyo psicológico $^{12,29}$ y educativo ${ }^{21,28,33}$, apreciaciones que coinciden con las propuestas de mejora verbalizadas por los entrevistados que además señalan como factor de ayuda el contacto con una asociación de afectados.

A modo de conclusión, se puede destacar la relevancia de una atención a aspectos psicosociales en el proceso asistencial de diálisis, desde una consideración de la influencia de la enfermedad y de sus tratamientos en la calidad de vida de los afectados. Además, el estudio revela la necesidad de una mejora de aspectos de carácter organizacional a través de una mayor homogeneidad, transparencia y eficacia en la gestión del proceso asistencial.

\section{BIBLIOGRAFÍA}

1. Organización Mundial de la Salud. Las enfermedades crónicas: la principal causa de mortalidad. www.who.int/chp/chronic_disease_report/part1/es/index1.html (citado 19/06/2007).

2. Prieto MA, Suess A, Escudero M, March JC. Las expectativas de los/as pacientes en 12 procesos asistenciales del sistema sanitario público de Andalucía. En: Consejería de Salud, Secretaría General de Calidad y Modernización (ed): Premios Investigación en Salud 2004. Sevilla: Consejería de Salud, Secretaría General de Calidad y Modernización 2005: 27-55.

3. Ponte PR, Conlin G, Conway JP, Grant S, MedeiRos C, NeIs J et al. Making patient-centered care come alive: achieving full integration of the patient's perspective. J Nurs Adm 2003; 33: 82-90.

4. HIBBARD JH. Engaging health care consumers to improve the quality of care. Med Care 2003; 41(Suppl. 70): 61-70.
5. Landreneau K, Lee K, Landreneau MD. Quality of life in patients undergoing hemodialysis and renal transplantation - A meta-analytic review. Nephrol Nurs J 2010; 37: 37-45.

6. KuRella Tamura M, Goldstein MK, Pérez-Stable EJ. Preference for dialysis withdrawal and engagement in advance care planning within a diverse simple of dialysis patients. Nephrol Dial Transplant 2010; 25: 237-242.

7. Kutner NG, Zhang R, Barnhart H, Collins AJ. Health status and quality of life reported by incident patients after 1 year on haemodialysis or peritoneal dialysis. Nephrol Dial Transplant 2005; 20: 2159-2167.

8. Helda K, Hartman A, Grootendorst DC, De Jager DJ, Leivestad T, Foss A et al. Benefit of kidney transplantation beyond 70 years of age. Nephrol Dial Transplant 2010; 25: 1680-1687.

9. Luongo M. The patient switching from HD to PD poses a clinical and emotional dilemma. Nephrol Nurs J 2004; 31: 590-591.

10. Wang T, Tzatt S, Dalglish C, Jassal SV, Bargman $\mathrm{J}$, VAS $\mathrm{S}$ et al. Peritoneal dialysis in the nursing home. Intern J Urology Nephrol 2002; 34: 405-408.

11. FowLER C, BAAS LS. Illness representations in patients with chronic kidney disesase on maintenance hemodialysis. Nephrol Nurs J 2006; 33: 173-186.

12. Ortega NS. Bienestar psicológico como factor de dependencia en hemodiálisis. Rev Enfermera 2002; 10: 17-20.

13. Ahmed S, Addicott C, Oureshi M, Pendleton N, Clague JE, Horan MA. Opinions of elderly people on treatment for end-stage renal disease. Gerontology 1999; 45: 156-159.

14. Hernández ME, Ocando A, Mora J, Lorenzo S, López K. Satisfacción del paciente en una unidad de hemodiálisis: Objetivo de calidad asistencial en enfermería. Rev Soc Esp Enferm Nefrol 2005; 8: 90-96.

15. Contreras MD, Rivero MF, Jurado MJ, Crespo R. Perfil actual del paciente en hemodiálisis hospitalaria. Análisis de sus necesidades. Rev Soc Esp Enferm Nefrol 2004; 7: 56-61.

16. Ismael MR, Bernardi C. Qualidades de vida de pessoas com doença renal crónica em tratamento hemodialítico. Rev Latino-am Enfermagem 2005; 13: 670-676.

17. NAVARRo J. La atención médica al paciente con nefropatía diabética: Excusatio non petita, acusatio manifesta. Nefrología 2007; 27: 109-113.

18. Albalate M, Caramelo C, Hernando L. Centros de diálisis extrahospitalaria: ¿de dónde ve- 
nimos, a dónde vamos? Nefrología 2007; 27: 114-117.

19. Alarcón C, Agullar OA, Jiménez A, Manrique CC. La calidad de vida en pacientes con trasplante renal, medida a través del índice de Karnofsky en un hospital general. Rev Asoc Mex Med Crít y Ter Int 2002; 16: 119-123.

20. Zimmermann PR, De Carvalho OJ, Mari JJ. Impacto da depressão e outros fatores psicossociais no prognóstico de pacientes renais crónicos. R Psiquiatr 2004; 26: 312-318.

21. Lies-Smith H. Percepciones y experiencias de los pacientes en prediálisis. EDTNAIERCA J 2005; XXXI: 134-137.

22. Martorelli A, Mustaca AE. Psicología positiva, salud y enfermos renales crónicos. Rev Nefrol Dial y Transpl 2004; 24: 99-104.

23. BaKewell AB, Higgins RM, Edmunds ME. Quality of life in peritoneal dialysis patients: Decline over time and association with clinical outcomes. Kidney Int 2002; 61: 239-248.

24. Ayanian JZ, Cleary PD, Weissman JS, Epstein AM. The effect of patients' preferences on racial differences in access to renal transplantation. NEJM 1999; 341: 1661-1670.

25. RodríGuez MA, Hernández D, GutiérRez MJ, Amer FJ, Calls J. Evaluación y manejo del dolor intradiálisis. Rev Soc Esp Enferm Nefrol 2006; 9: 65-70.

26. Morelon E, Berthoux F, Brun-Strang C, Fior S, Volle R. Partners' concerns, needs and expectations in ESRD: results of the CODIT Study. Nephrol Dial Transplant 2005; 20: 16701675.

27. Pruchno RA, Lemay EP, Field L, Levinsky NG. Spouse as Health Care Proxy for Dialysis $\mathrm{Pa}-$ tients: Whose Preferences Matter? Gerontologist 2005; 45: 812-819.

28. Bañobe A, Vázquez J, Outeiriño S, Rodríguez M, González M, Graña J et al. Efectividad de la intervención educativa en cuidadores de pacientes dependientes en diálisis y valoración de la carga. Rev Soc Esp Enferm Nefrol 2005; 8: 156-165.

29. Carretero C, García ai, Gutiérrez M, Hilara L, Marcos L, Gallar P et al. Aplicación de la telemedicina en diálisis peritoneal. Rev Soc Esp Enferm Nefrol 2004; 7: 65.

30. Blalock S, Gómez N. What is better for Patients and Staff - Independent Units or National Dialysis Chains. Nephrol Nurs J 2006; 33 : 81-82.
31. BohIGAS L. La coordinación entre Atención Primaria y Especializada, clave para mejorar la calidad de la Nefrología. Nefrología 2003; 10: 7-9.

32. Saudan P, Halabi G, Perneger T, Wasserfallen JB, Kossovsky M, Feldman J. Variability in quality of care among dialysis units in western Switzerland. Nephrol Dial Transplant 2005; 20: 1854-1863.

33. Balovlenkov EK. Product Selection - Who Decides? Nephrol Nurs J 2004; 31: 354.

34. Heimburger O, Mujais S. Buffer transport in peritoneal dialysis. Kidney International 2003; 64 (Suppl 88): S37-S42.

35. Pifer TB, Bragg-Gresham JL, Dykstra DM, Shapiro JR, OPpenheimer CC, Gaylin DS et al. Quality of life and patient satisfaction: ESRD managed care demonstration. HCFR 2003; 24: 45-58.

36. МаскRETH R. Developing an on-site dialysis treatment center. Nursing Homes 2001; 50 (9): 21-31.

37. Cirera F, Reina M. Valoración de la información sobre el trasplante renal que reciben los pacientes en los centros periféricos de hemodiálisis. Rev Soc Enferm Nefrol 2005; 8: 64-74.

38. AbRIL MD, Iglesias R, Jerez A, López MJ, MaÑé N, YUSTE E. Aplicación de una técnica cualitativa para la elaboración de un plan de atención al paciente pluripatológico en hemodiálisis. Rev Soc Esp Enferm Nefrol 2004; 7: 275-279.

39. Carretero C, García al, Gutiérrez M, Hilara L, Marcos L, Gallar P et al. Aplicación de la telemedicina en diálisis peritoneal Rev Soc Esp Enferm Nefrol 2004; 765.

40. Carreras RM, Cerrillo V, Agramunt I, Aicart C, Baldayo B, Carratalá J et al. Urgencias en diálisis en toda una provincia. Rev Soc Esp Enferm Nefrol 2006; 9: 146-147.

41. March JC, Prieto MA, Hernán M, Solas O. Técnicas cualitativas para la investigación en salud pública y gestión de servicios de salud: algo más que otro tipo de técnicas. Gac Sanit 1999; 13: 312-319.

42. Parasuraman A, Zeithaml VA, Berry LL. SERVQUAL: A multiple item scale for measuring consumer perceptions of quality service. $\mathrm{J}$ Retail 1988; 64: 12-40.

43. Prieto MA, March JC, Suess A, Ruiz A, Terol E, CASAl J. Percepción de los pacientes sobre la seguridad de la asistencia sanitaria. Med Clin (Barc) 2008: 131: 33-38. 